\title{
Differential Search Algorithm-based Parametric Optimization of Fuzzy Generalized Eigenvalue Proximal Support Vector Machine
}

\author{
M. H. Marghny \\ Computer Science Department, \\ Faculty of Computers and \\ Information, Assiut University, \\ Egypt.
}

\author{
Rasha M. Abd El-Aziz \\ Computer Science Department, \\ Faculty of Science, Assiut \\ University, Egypt.
}

\author{
Ahmed I. Taloba \\ Computer Science Department, \\ Faculty of Computers and \\ Information, Assiut University, \\ Egypt
}

\begin{abstract}
Support Vector Machine (SVM) is an effective model for many classification problems. However, SVM needs the solution of a quadratic program which require specialized code. In addition, SVM has many parameters, which affects the performance of SVM classifier. Recently, the Generalized Eigenvalue Proximal SVM (GEPSVM) has been presented to solve the SVM complexity. In real world applications data may affected by error or noise, working with this data is a challenging problem. In this paper, an approach has been proposed to overcome this problem. This method is called DSA-GEPSVM. The main improvements are carried out based on the following: 1) a novel fuzzy values in the linear case. 2) A new Kernel function in the nonlinear case. 3) Differential Search Algorithm (DSA) is reformulated to find near optimal values of the GEPSVM parameters and its kernel parameters. The experimental results show that the proposed approach is able to find the suitable parameter values, and has higher classification accuracy compared with some other algorithms.
\end{abstract}

\section{Keywords}

Support Vector Machines, Generalized Eigenvalues, Proximal Classifier, Fuzzy Data Classification, Differential Search Algorithm, Kernel Function.

\section{INTRODUCTION}

Recently, information, growing in huge volumes creates the need to process large amounts of data. In order to find hidden patterns of data and convert them into useful knowledge, this is known Data Mining. This direction includes methods other than classical analysis, based on clustering analysis [1-4], classification analysis [5, 6], and solving problems of generalization, association and finding patterns [7-9]. This area of research has recently become more and more important.

Classification is the process of arranging data into homogenous group or classes according to some common characteristics present in the data. Support vector machine (SVM) has an excellent performance in many real life classification problems such as image processing, text classification and bioinformatics.

SVM which is an emerging data classification technique proposed by Vapnik in 1995 [10], and has been widely adopted in various fields of classification, nevertheless it suffers from complexity and parameters selection. A new method has been introduced in [11] by Olvi L. Mangasarian which called Proximal Support Vector Machine (PSVM). This method has solved the problem of complexity of standard SVM, but it suffers from poor performance in the case of noisy and unbalanced data. Recently an efficient approach to
PSVM has been proposed also by Olvi L. Mangasarian which is called the Generalized Eigenvalue Proximal Support Vector Machine (GEPSVM) [12]. The complexity of standard support vector machine has been solved by GEPSVM. A fundamental difference between GEPSVM and SVM is that, GEPSVM solves two generalized eigenvalue problems to obtain two non-parallel hyper-planes, whereas, SVM solves one quadratic programming problem (QPP) to obtain one hyper-plane. Therefore, GEPSVM works faster than SVM. Experimental results in [12] showed the effectiveness of GEPSVM on some public datasets.

In real world applications data may affected by noise or error which significantly influences on the performance of GEPSVM. There are many approaches have been proposed by researchers for this problem [13-19]. More efforts are needed in order to improve the performance of the classification task in this type of data.

In addition, the major problems that are encounter in SVM and all its inferred methods are how to find near optimal values for the SVM parameters and select a SVM kernel as well as tuning its parameters. Unsuitable parameters setting lead to poor classification outcomes. Authors in [20-25] tried to find solution for SVM parameters. There are no particular method to find the optimal values for the SVM or GEPSVM parameters and kernel parameters. This problem is still an interesting topic for more research to find more appropriate values for GEPSVM parameters and kernel parameters.

For these reasons, an improved version of GEPSVM, called DSA-GEPSVM for short is proposed. A new method for computing the fuzzy membership function is used in the linear case. Furthermore, a new kernel is used in the nonlinear case. The new kernel is a combination between the polynomial and the radial base function kernel. For solving the problem of parameters selection, a new and powerful method which is called Differential Search Algorithm (DSA) [26-31] has been used. This makes the optimal separating hyper-planes obtainable in both linear and non-linear classification problems.

The remainder of this paper is organized as follows. In section 2 we briefly give description of the GEPSVM. Section 3 give description of the DSA. In section 4 the proposed method is described. Section 5 reports experimental results. Finally, the conclusions make up Section 6.

2. GENERALIZED EIGENVALUE PSVM In 2006 Olvi L. Mangasarian and Edward W. Wild proposed the Generalized Eigenvalue Proximal Support Vector Machine GEPSVM [12] as a generalization of the SVM method. The new formulation does not need the planes to be parallel, but for each class, the algorithm finds a plane that is 
as close as possible to the points of one class and as far as possible to those in the other class. Due to the simplicity of GEPSVM, many researchers have refined it to improve the general performance of the classifier [32-35]. But GEPSVM still needs more improvements, and is a very good topic for researchers.

First we consider the classification problem of $m$ points in the $\mathrm{n}$ dimensional real space $R^{n}$, represented by the $m_{1} \times n$ matrix A belonging to class 1 and $m_{2} \times n$ matrix $\mathrm{B}$ belonging to class 2 , with $m_{1}+m_{2}=m$. For this problem, a standard linear SVM is given by a plane halfway between the two parallel bounding planes that bound two disjoint half spaces each containing points mostly of class 1 or 2 [12].

In MSPSVM the parallelism condition has been dropped, but requires that each plane be as close as possible to one of the data sets and as far as possible from the other one. Thus, we are seeking for the two planes in $R^{n}$ :

$P 1: x^{\prime} w^{1}-\gamma^{1}=0, P 2: x^{\prime} w^{2}-\gamma^{2}=0$,

where the plane $P 1$ is closest to the points of class 1 and furthest from the points in class 2 , while the plane $P 2$ is closest to the points in class 2 and furthest from the points in class 1 . Then the first plane of (1) is obtained by solving the following optimization problem:

$\min _{(w, \gamma) \neq 0} \frac{\|A w-e \gamma\|^{2} /\left\|\left[\begin{array}{l}w \\ \gamma\end{array}\right]\right\|^{2}}{\|B w-e \gamma\|^{2} /\left\|\left[\begin{array}{l}w \\ \gamma\end{array}\right]\right\|^{2}}$,

where $\|$.$\| is the two-norm, and it has been assumed in [12]$ that $(w, \gamma) \neq 0$ which implies to $B w-e \gamma \neq 0$. As introduced in [12] the numerator of the minimization problem (2) is the sum of squares of two-norm distances in the $(w, \gamma)$-space of points in the first class to the plane $x^{\prime} w^{1}-\gamma^{1}=0$, while the denominator of (2) is the sum of squares of two norm distances in the $(w, \gamma)$ space of points in the second class to the same plane. By simplifying (2) we can write,

$\min _{(w, \gamma) \neq 0} \frac{\|A w-e \gamma\|^{2}}{\|B w-e \gamma\|^{2}}$.

Then Tikhonov regularization term is added [36] to reduce the norm of the problem variables $(w, \gamma)$ that determine the proximal planes (1). Thus, for a parameter $\delta$, problem (3) has been rewritten as follows:

$\min _{(w, \gamma) \neq 0} \frac{\|A w-e \gamma\|^{2}+\delta\left\|\left[\begin{array}{c}w \\ \gamma\end{array}\right]\right\|^{2}}{\|B w-e \gamma\|^{2}}$,

We can rewrite (4) as follow:

$\min _{z \neq 0} r(z):=\frac{z^{\prime} G z}{z^{\prime} H z}$,

(5)

where,

$G:=\left[\begin{array}{ll}A & -e\end{array}\right]^{\prime}\left[\begin{array}{ll}A & -e\end{array}\right]+\delta I$,

$H:=\left[\begin{array}{ll}B & -e\end{array}\right]^{\prime}\left[\begin{array}{ll}B & -e\end{array}\right], \quad z:=\left[\begin{array}{l}W \\ \gamma\end{array}\right]$.

$G$ and $H$ are symmetric matrices in $R^{(n+1) \times(n+1)}$ and $I$ is an identity matrix.

As pointed out in [37], the objective function of (5) is known as the Rayleigh quotient, hence its solution can be obtained by solving a generalized eigenvalues problem. That is, the eigenvector corresponding to the smallest eigenvalue can determine a plane effectively.
Similarly we can directly get the second plane by solving the following optimization problem.

$\min _{z \neq 0} s(z):=\frac{z^{\prime} L z}{z^{\prime} M z}$

(8)

where,

$L:=\left[\begin{array}{ll}B & -e\end{array}\right]^{\prime}\left[\begin{array}{ll}B & -e\end{array}\right]+\delta I$,

$M:=\left[\begin{array}{ll}A & -e\end{array}\right]^{\prime}\left[\begin{array}{ll}A & -e\end{array}\right]$.

$\mathrm{L}$ and $\mathrm{M}$ are again symmetric matrices in $R^{(n+1) \times(n+1)}$. As analyzed above, the two non-parallel planes can be obtained directly by solving the classical generalized eigenvalue problem.

The Nonlinear GEPSVM can be obtained easily by considering the problem of finding two non-parallel planes

P1: $K\left(x^{\prime}, C^{\prime}\right) u^{1}-\gamma^{1}=0, P 2: K\left(x^{\prime}, C^{\prime}\right) u^{2}-\gamma^{2}=0$,

where $C:=\left[\begin{array}{l}A \\ B\end{array}\right]$.

$\mathrm{K}$ is the kernel function, which will be presented in the next section. By employing the same regularization strategy as in equations (4-10), we can also obtain the two non-parallel planes by solving the optimization problems of the following equations:

$\min _{z \neq 0} r(z):=\frac{z^{\prime} G z}{z^{\prime} H z}, \quad$ where $z:=\left[\begin{array}{l}u \\ \gamma\end{array}\right]$,

$\min _{z \neq 0} s(z):=\frac{z^{\prime} L z}{z^{\prime} M z}, \quad$ where $z:=\left[\begin{array}{l}u \\ \gamma\end{array}\right]$,

(13)

where,

$G:=\left[\begin{array}{ll}K\left(A, C^{\prime}\right) & -e\end{array}\right]^{\prime}\left[K\left(A, C^{\prime}\right)-e\right]+\delta I$,

$H:=\left[\begin{array}{ll}K\left(B, C^{\prime}\right) & -e\end{array}\right]^{\prime}\left[K\left(B, C^{\prime}\right)-e\right]$,

$L:=\left[K\left(B, C^{\prime}\right)-e\right]^{\prime}\left[K\left(B, C^{\prime}\right)-e\right]+\delta I$,

$M:=\left[K\left(A, C^{\prime}\right) \quad-e\right]^{\prime}\left[K\left(A, C^{\prime}\right) \quad-e\right]$.

$\mathrm{G}, \mathrm{H}, \mathrm{L}$ and $\mathrm{M}$ are symmetric matrices in $R^{(m+1) \times(m+1)}$.

Since 2006 GEPSVM has achieved great performance in many real live applications, but in some cases data may affected by noise and errors. Most classification methods give low classification accuracy with this kind of data, and need some modifications in order to increase the classification accuracy. One of the most effective ways to overcome this problem is by adding a fuzzy value to each training sample. The works of many researches carried out by adding fuzzy values to the standard SVM [13,14, 17, 18]. Many attempts for adding fuzzy to GEPSVM have been illustrated as in [15, 16, 19]. A first attempt to obtain a fuzzy version of the GEPSVM classification is presented in $[15,16]$. In [15] the authors attempt to solve the following problem:

$\min _{(w, \gamma) \neq 0} \frac{\left\|S^{A} A w-e \gamma\right\|^{2}}{\left\|S^{B} B w-e \gamma\right\|^{2}}$.

With $S^{A}$ is the fuzzy membership weights for each point $A_{i}$ and $S^{B}$ is the fuzzy membership weights for each point $B_{i} \cdot S^{A}$ and $S^{\mathrm{B}}$ are diagonal matrices.

$S_{i i}^{A}=0.5+\frac{e^{f\left(d\left(A_{i}, C_{B}\right)-d\left(A_{i}, C_{A}\right)\right) / d_{A B}-e^{-f}}}{2\left(e^{f}-e^{-f}\right)}, i=1, \ldots, p$, 
$S_{i i}^{B}=0.5+\frac{e^{f\left(d\left(B_{i}, C_{A}\right)-d\left(B_{i}, C_{B}\right)\right) / d} A B-e^{-f}}{2\left(e^{f}-e^{-f}\right)}, i=1, \ldots, m$.

Where $C_{A}$ and $C_{B}$ are the center of mass of the two classes, $\mathrm{d}_{\mathrm{AB}}$ is the distance between the two means, the function $d(.,$. is the Euclidean distance between two points, and $\mathrm{f}$ is a constant that determines the rate at which the fuzzy membership decreases towards 0.5 . Another recently attempt of fuzzy GEPSVM can be found in [19] where the author proposed the following fuzzy function

$S_{i i}^{A}=s+(1-s) \cdot e^{-\left(\frac{\min \mathbb{\operatorname { m a x }} \operatorname{ma}\left(A_{i}, C_{A}\right.}{\operatorname{mad}\left(A_{i}, C_{B}\right.}\right)^{2}}$.

Where $\min \left(d\left(A_{i}, C_{A}\right)\right)$ is the minimum distance of the point $A_{i}$ from the centers in $C_{A}, \max \left(d\left(A_{i}, C_{B}\right)\right)$ is the maximum distance of the point $A_{i}$ from the centers $C_{B}$ of the other class, and $\mathrm{s}$ is a parameter weighting the contribution of the exponential term to $S^{A}$, for more detail see [19].

\section{DIFFERENTIAL SEARCH ALGORITHM (DSA)}

Differential Search Algorithm (DSA) is a recently and efficient evolutionary algorithm. DSA is effectively used to solve numerical optimization problems. The main idea of the DSA algorithm was inspired form the migration of superorganisms making use of brownian like motion [28].

Algorithms that make use of the principle of evolutionary computation are known as Evolutionary Algorithms (EA). These algorithms are suitable to search for the optimal (best) solution of many optimization problems. In real world problems the optimization process may have more than one solution, for searching for the optimal solution among all these solutions in a short time is a challenging task. If the search space is small then searching for the optimal solution will take short time. Working with data whose search space is very large is a challenge for most researchers. When the problem is very large with a great number of possible solutions, then finding the optimal solution is difficult. Evolutionary computation techniques are powerful and effective with this kind of data. EA includes the following techniques [28]:

- Ant colony algorithm

- Artificial Bee Colony (ABC) algorithm

- Cultural algorithms

- Differential evolution

- Evolutionary algorithms

- Evolutionary programming

- Evolution strategy

- Gene expression programming

- Genetic algorithm

- Genetic programming

- Harmony search

- Learnable Evolution Model

- Particle swarm optimization

- Self-organization such as self-organizing maps

- Swarm intelligence

The Differential Search Algorithm (DSA) is the most recent addition. There are a number of computational-intelligence algorithms that model the behaviors of the superorganisms [28-30]. In the present work DSA is used to get the best values of parameter values in the proposed algorithm, due to it has the ability to manage such problem. The pseudo-code indicating the function of DS algorithm is given in Appendix.

\section{PROPOSED APPROACH}

\subsection{Linear Fuzzy DSA-GEPSVM}

The proposed approach introduce a technique for computing the fuzzy membership values. If the data affected with noise or outliers then the classification process will influence, so the data needs some preprocessing steps. We propose a method by adding a fuzzy value for those examples that away from the center of the class and the remaining examples don't have any change. Now the new formulation of the problems become as follow:

$\min _{(w, \gamma) \neq 0} \frac{\left\|S^{A} A w-e \gamma\right\|^{2}+\delta\left\|\left[\begin{array}{l}w \\ \gamma\end{array}\right]\right\|^{2}}{\left\|S^{B} B w-e \gamma\right\|^{2}}$,

where,

$G:=\left[\begin{array}{ll}S^{A} A & -e\end{array}\right]^{\prime}\left[\begin{array}{ll}S^{A} A & -e\end{array}\right]+\delta I$,

$H:=\left[\begin{array}{ll}S^{B} B & -e\end{array}\right]^{\prime}\left[\begin{array}{ll}S^{B} B & -e\end{array}\right], \quad z:=\left[\begin{array}{l}W \\ \gamma\end{array}\right]$.

The optimization problem (22) becomes:

$\min _{z \neq 0} r(z):=\frac{z^{\prime} G z}{z^{\prime} H z}$.

(25)

Similarly we can directly get the second fuzzy plane by solving the following optimization problem.

$\min _{z \neq 0} s(z):=\frac{z^{\prime} L z}{z^{\prime} M z}$

(26)

where,

$L:=\left[\begin{array}{ll}S^{B} B & -e\end{array}\right]^{\prime}\left[\begin{array}{ll}S^{B} B & -e\end{array}\right]+\delta I$,

$M:=\left[\begin{array}{ll}S^{A} A & -e\end{array}\right]^{\prime}\left[\begin{array}{ll}S^{A} A & -e\end{array}\right]$.

Figure 1 explains the process of computing fuzzy matrix $\mathrm{S}^{\mathrm{A}}$.

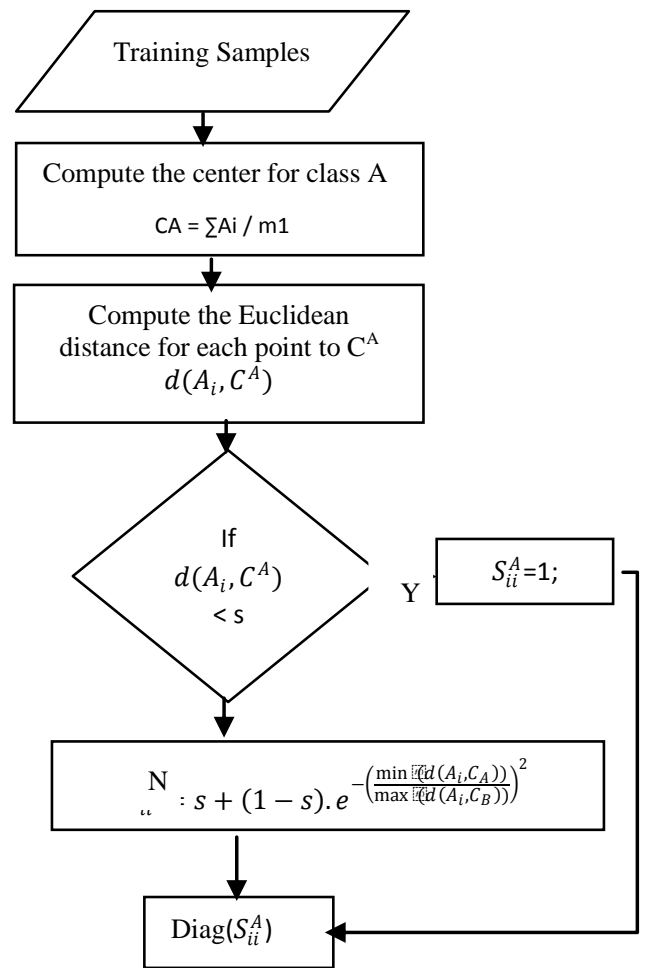

Fig 1: Methodology of calculation of fuzzy matrix $S^{A}$ 
Where $\mathrm{s}$ is a parameter weighting the contribution of the exponential term to $S^{A}$. The same way for $S^{B}$. The next step and the most important step in the proposed method is how we can get the optimal parameters. In the linear MSPSVM there is only one parameter $\delta$. DSA algorithm has been used to find the optimal value of $\delta$.

\subsection{Nonlinear DSA-GEPSVM}

Appropriate choice of the kernel function increases the accuracy of the classification. In real life applications the choice of kernel function depends on the dataset used.

Here are some of the most popular kernels.

Polynomial function:

A polynomial kernel is a common method for nonlinear modeling.

$\left.K\left(x, x^{\prime}\right)=\left(<x, x^{\prime}\right\rangle+1\right)^{d}$.

Gaussian radial basis function:

This function has received significant attention, most commonly with a Gaussian of the form,

$K\left(x, x^{\prime}\right)=\exp \left(\frac{-\left\|x-x^{\prime}\right\|^{2}}{2 \sigma^{2}}\right)$.

Exponential radial basis function:

$K\left(x, x^{\prime}\right)=\exp \left(\left(-\left\|x-x^{\prime}\right\|\right) /(D \sigma)\right)$.

In [38] a new kernel has been introduced the author used the new kernel with the standard SVM, in the presented work we use the new kernel which is called PolyRBF witch is a hybrid between a polynomial kernel and a Gaussian RBF kernel.

$K\left(x, x^{\prime}\right)=\left(1+\exp \left(\frac{-\left\|x-x^{\prime}\right\|^{2}}{D \sigma}\right)\right)^{d}$.

Where $\mathrm{D}$ is the dimension of the data, now we have three parameters in the nonlinear case, the first parameter is the regularization of GEPSVM and second and third parameters is for the kernel function if we use the polynomial kernel then we have the parameter $\mathrm{d}$, and if we use the RBF then we have the parameter $\sigma$, last if we use the PolyRBF the we have two parameters $\mathrm{d}$ and $\sigma$.

In order to get the nonlinear planes in equation (11), we proposed to use the PolyRBF kernel. In the next subsection we explain how to get the best parameters in the linear and nonlinear classifier.

\subsection{Parameter Optimization using DSA}

A population in DSA assumed to be made up of random solutions of the problem corresponds to an artificialsuperorganism migrating. In DSA, artificial- superorganism migrates to the global minimum value of the optimization problem. In the migration time the artificial-superorganism tests whether some positions which was selected randomly are suitable temporary during the migration. Then the process stops over on the suitable tested position for a temporary time during the migration, the members of the artificial that made such discovery immediately settle at the discovered position and continue their migration from this position [28].

In the implementation of DSA, artificial-organisms (i.e., $\mathrm{X}_{\mathrm{i}}$, $\mathrm{i}=\{1,2,3, \ldots, \mathrm{N}\})$ making up an artificial-superorganism (i.e., Superorganismg, $\mathrm{g}=\{1,2,3, \ldots, \mathrm{G}\})$ contain members as much as the size of the problem (i.e., $\mathrm{x}_{\mathrm{ij}}, \mathrm{j}=\{1,2,3, \ldots, \mathrm{D}\}$ ). Where, $\mathrm{N}$ signifies number of elements in the superorganism (Size of the population), $\mathrm{G}$ represents number of maximum generation, and $\mathrm{D}$ indicates size of the problem [28-30]. $x_{i j}=\operatorname{rand} .\left(u p_{j}-\operatorname{low}_{j}\right)+$ low $_{j}$

The stopover site is an important step in migration. The method to find a stopover site at the remaining between the artificial- organisms may be described by a Brownian-like random walk model [28]. By a random selection of individuals of the artificial- organisms move toward the targets of donor $=\left[X_{\text {random shuffling (i) }}\right]$ to discover stopover sites. The scale value $(\mathrm{R})$ is used to control the size of the change occurred in the positions of members of the artificialorganisms. The way of calculation $\mathrm{R}$ makes the respective artificial-superorganism to radically change direction in the habitat [28-30].

The stopover site position in DSA is produced by using equation (34):

StopoverSite $=$ Superorganism + Scale $*($ donor Superorganism)

A random process is used to determine the members of the artificial organisms of the superorganism of stopover site. If the one of the stopover site elements goes outside the limits of the search space for any reason, it randomly deferred to another position in the search space. If the stopover site is better than the sources owned by the artificial-organism, the artificial-organism moves to that stopover site. While the artificial-organisms change site, the superorganism containing the artificial organisms continues its migration to the global minimum.

There are two control parameters in DSA, which are p1 and p2. The tested and the most appropriate values for these parameters were conducted by [28]. Figure 2 describes the main steps of the proposed approach.

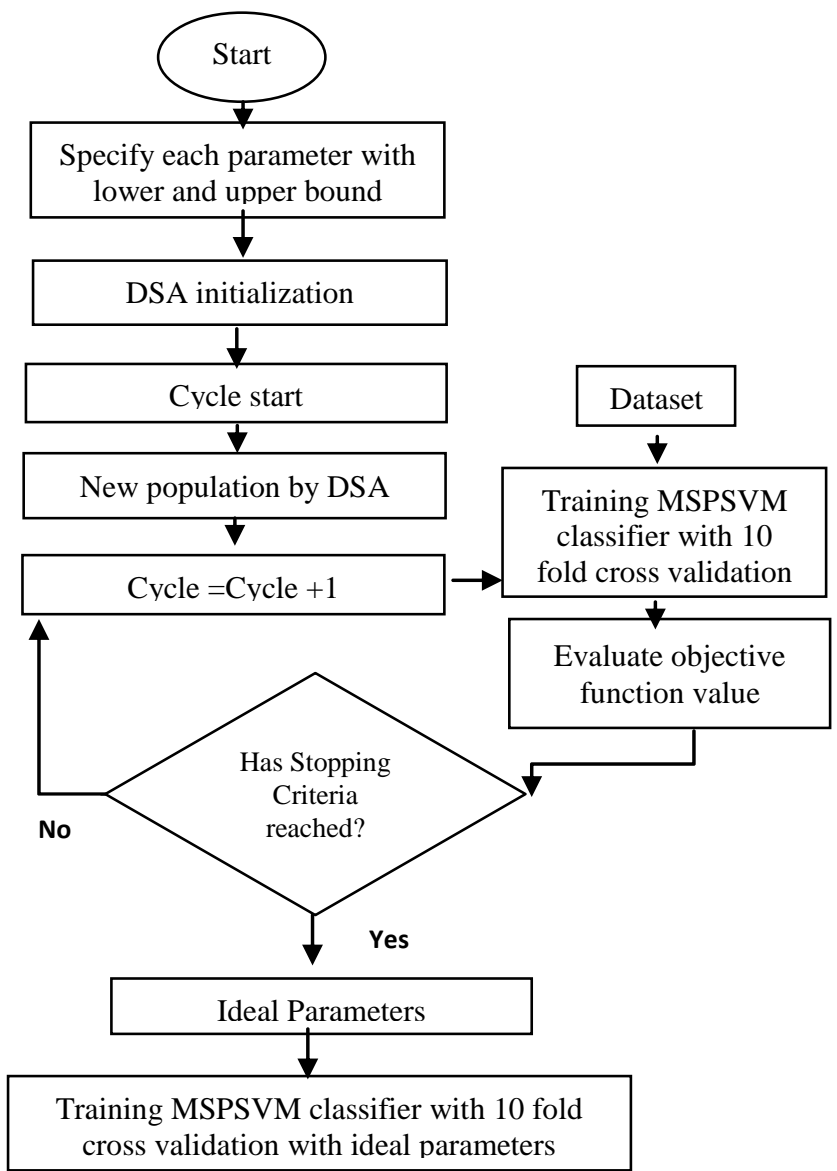

Fig 2: Basic steps of the proposed approach 
In the proposed approach we are seeking for optimal values for the linear and nonlinear parameters. The stopping criteria for the procedure is either max number of cycles are reached or the $100 \%$ accuracy is obtained.

\section{EXPERIMENTAL RESULTS}

The proposed approach is implemented on personal computer with a core i3 processor $2.13 \mathrm{GHz}, 3 \mathrm{~GB}$ of RAM, and windows 8.1 operating system. Matlab 2010b framework is used in development. To verify the proposed approach quality the following datasets are used from UCI repository [39], the datasets characteristics are shown in Table 1.

Table 1 Datasets characteristics.

\begin{tabular}{|c|c|c|}
\hline Dataset & Features & Instances \\
\hline Australian & 14 & 690 \\
\hline Breast Cancer & 10 & 683 \\
\hline Diabetes & 8 & 768 \\
\hline German & 24 & 1000 \\
\hline Heart Disease & 13 & 270 \\
\hline Ionosphere & 34 & 351 \\
\hline Liver Disorders & 6 & 345 \\
\hline Sonar & 60 & 208 \\
\hline Splice & 60 & 1000 \\
\hline Wbpc & 34 & 198 \\
\hline
\end{tabular}

Tables 2 and 6 summarize all parameters setting in the linear and nonlinear DSA-MSPSVM respectively with their assigned values. Where the values are chosen based on our numerical experiments.

For implementation, the data was divided into ten parts or folds, nine of which comprised the training data, with the tenth being used for testing the generalization ability of the classifier. This process was repeated ten times, using a different fold for testing on each occasion. This process is known as tenfold cross validation and is a standard methodology for reporting the performance of a classifier. The classification accuracy was computed by computing the average across all the ten trials.

Table 2 Linear GEPSVM parameter

\begin{tabular}{|c|c|c|}
\hline Parameter & Symbol & Interval \\
\hline P1 & $\delta$ & {$[0.001,10000]$} \\
\hline
\end{tabular}

The DSA parameters setting are shown in table 3.

Table 3 DSA-GEPSVM parameters setting

\begin{tabular}{|c|c|c|}
\hline DSA Parameter & Definition & Value \\
\hline Popsize & Size of superorganism & 30 \\
\hline Dim & Dimension of search space & 1 \\
\hline Low & Minimum limit of search space & 0.001 \\
\hline Up & Maximum limit of search space & 10000 \\
\hline Maxcycle & Max number of Iteration & 20 \\
\hline
\end{tabular}

Table 4 illustrates the results obtained after implementing Linear DSA-GEPSVM on several public benchmark datasets.

Table 4 Linear DSA-GEPSVM accuracy results on some benchmark datasets

\begin{tabular}{|c|c|c|c|}
\hline \multirow{2}{*}{ Dataset } & \multicolumn{3}{|c|}{ Proposed method } \\
\cline { 2 - 4 } & P1 & Training & Testing \\
\hline Australian & 920.9123 & 70.6924 & 73.913 \\
\hline Breast Cancer & 4.8162 & 97.3984 & 100 \\
\hline Liver Disorders & 964.9806 & 70.7395 & 77.1429 \\
\hline Diabetes & 5 & 74.2775 & 80.5195 \\
\hline German & 15.8707 & 74.7778 & 82 \\
\hline Heart Disease & 0.8947 & 87.2428 & 96.2963 \\
\hline Ionosphere & 0.1306 & 81.132 & 76.064 \\
\hline Sonar & 0.2105 & 91.9786 & 90.4762 \\
\hline Splice & 40.3143 & 74.8889 & 76 \\
\hline wpbc & 0.001 & 89.3258 & 95 \\
\hline
\end{tabular}

Table 5 illustrates the comparison between Linear DSAGEPSVM and four recently methods GEPSVM, FSVM, FTSVM, and IGEPSVM. The proposed method given promising results for all dataset from other methods, and the mean accuracy of proposed method is the best.

Table 5 Training accuracy of linear DSA-GEPSVM and compared methods on UCI datasets

\begin{tabular}{|c|c|c|c|c|c|}
\hline Dataset & Proposed & $\begin{array}{c}\text { GEPSVM } \\
{[\mathbf{1 2}]}\end{array}$ & $\begin{array}{c}\text { FSVM } \\
{[\mathbf{1 3}]}\end{array}$ & $\begin{array}{c}\text { FTSVM } \\
{[\mathbf{1 8}]}\end{array}$ & $\begin{array}{c}\text { IGEPSVM } \\
{[\mathbf{3 6}]}\end{array}$ \\
\hline Australian & 70.6924 & - & 85.56 & $\mathbf{8 6 . 0 8}$ & - \\
\hline Breast Cancer & $\mathbf{9 7 . 3 9 8 4}$ & - & 65.01 & 65.60 & - \\
\hline Liver Disorders & 70.7395 & 68.86 & 76.67 & $\mathbf{7 7 . 8 0}$ & 73.83 \\
\hline Diabetes & 74.2775 & 67.93 & - & - & $\mathbf{7 4 . 6 1}$ \\
\hline German & 74.7778 & 75.49 & 71.68 & $\mathbf{7 8 . 2 0}$ & 77.15 \\
\hline Heart Disease & $\mathbf{8 7 . 2 4 2 8}$ & - & 83.33 & 84.44 & - \\
\hline Sonar & $\mathbf{9 1 . 9 7 8 6}$ & 83.66 & - & - & 88.47 \\
\hline wpbc & $\mathbf{8 9 . 3 2 5 8}$ & 83.98 & - & - & 87.74 \\
\hline mean & $\mathbf{8 2 . 0 5 4 1}$ & 75.984 & 79.31 & 78.424 & 80.36 \\
\hline
\end{tabular}

The deferent kernels were applied to nonlinear DSAGEPSVM. The first kernel is the polynomial kernel, the second kernel is the radial base function kernel and the last one the hybrid kernel between the previously mentioned kernels. In order to prove how the hybrid kernel is effective, we applied the nonlinear DSA-GEPSVM three times on each kernel. Table 7 shows the detailed results that obtained. Figure 3 shows how the hybrid kernel is effective in most cases. 
Table 6: Nonlinear DSA-GEPSVM parameters

\begin{tabular}{|c|c|c|}
\hline Parameter & Symbol & Interval \\
\hline P1 & $\delta$ & {$[0.001,10000]$} \\
\hline P2 & $\sigma$ & {$[0.001,33]$} \\
\hline P3 & $\mathrm{p}$ & {$[0.001,33]$} \\
\hline
\end{tabular}

Table 7: The nonlinear DSA-GEPSVM results applied to the three mentioned kernels

\begin{tabular}{|c|c|c|c|c|c|c|c|}
\hline Dataset & Kernel & P1 & P2 & P3 & $\begin{array}{c}\text { ACC } \\
\text { Training }\end{array}$ & $\begin{array}{c}\text { ACC } \\
\text { Testing }\end{array}$ & $\begin{array}{c}\text { ACC } \\
\text { Breast }\end{array}$ \\
Cancer & poly & 1000.0000 & - & 4.5906 & 100.00 & 100.00 & 100.00 \\
& PolyRBF & 125.4612 & 24.8243 & 5.3731 & 96.92 & 98.55 & 97.73 \\
\hline \multirow{3}{*}{ Heart } & rbf & 0.0010 & 4.5501 & - & 87.24 & 88.89 & 88.07 \\
& poly & 1000.0000 & - & 8.9368 & 100.00 & 81.48 & 90.74 \\
& PolyRBF 2516.2190 & 12.9724 & 25.134 & 100.00 & 85.19 & 92.59 \\
\hline \multirow{5}{*}{ Ionosphere } & Poly & 0.0010 & - & 4.1069 & 100.00 & 80.00 & 90.00 \\
& PolyRBF & 841.7760 & 32.5201 & 24.1597 & 100.00 & 97.06 & 98.53 \\
\hline Sonar & rbf & 13.1398 & 0.001 & - & 100.00 & 90.00 & 95.00 \\
& poly & 8884.2091 & - & 6.3464 & 100.00 & 76.19 & 88.10 \\
& PolyRBF & 0.0010 & 19.9701 & 19.631 & 100.00 & 85.71 & 92.86 \\
\hline
\end{tabular}

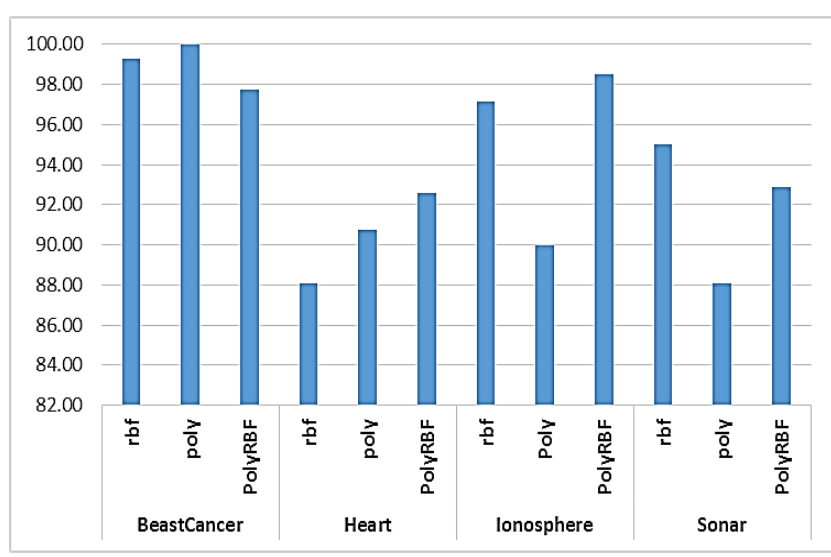

Fig 3: Mean accuracies of poly, rbf, and PolyRBF applied on UCI datasets

Table 8 and Table 9 illustrate the comparison between training and testing accuracies of nonlinear DSA-GEPSVM on four datasets with other recently methods GA+SVM, $\mathrm{SA}+\mathrm{SVM}, \mathrm{PSO}+\mathrm{SVM}, \mathrm{CV}-\mathrm{ACC}$, and S.C. Chen. Results proved how the proposed approach give comparable and promising results.

Table 8: Training accuracies comparisons between the nonlinear DSA-GEPSVM with PolyRBF and other recently approaches

\begin{tabular}{|c|c|c|c|c|}
\hline \multirow{2}{*}{ Method } & \multicolumn{4}{|c|}{ Datasets } \\
\cline { 2 - 5 } & $\begin{array}{c}\text { Breast } \\
\text { Cancer }\end{array}$ & $\begin{array}{c}\text { Heart } \\
\text { Disease }\end{array}$ & Ionosphere & Sonar \\
\hline Proposed & 96.92 & $\mathbf{1 0 0}$ & $\mathbf{1 0 0}$ & $\mathbf{1 0 0}$ \\
\hline
\end{tabular}

\begin{tabular}{|c|c|c|c|c|}
\hline GA+SVM[20] & 94.23 & 94.58 & 96.61 & 95.22 \\
\hline SA+SVM[21] & $\mathbf{9 7 . 9 5}$ & 87.97 & 97.5 & 91.85 \\
\hline PSO+SVM[22] & $\mathbf{9 7 . 9 5}$ & 88.17 & 97.5 & 88.32 \\
\hline CV-ACC[23] & 96.69 & 84.753 & 97.714 & $\mathbf{1 0 0}$ \\
\hline S.C. Chen [24] & 96.04 & 86.32 & 96.60 & 96.07 \\
\hline
\end{tabular}

Table 9: Testing accuracies comparisons between the nonlinear DSA-GEPSVM with PolyRBF and other recently approaches

\begin{tabular}{|c|c|c|c|c|}
\hline \multirow{2}{*}{ Method } & \multicolumn{4}{|c|}{ Datasets } \\
\cline { 2 - 5 } & $\begin{array}{c}\text { Breast } \\
\text { Cancer }\end{array}$ & $\begin{array}{c}\text { Heart } \\
\text { Disease }\end{array}$ & Ionosphere & Sonar \\
\hline Proposed & $\mathbf{9 8 . 5 5}$ & 85.19 & 97.06 & 85.71 \\
\hline GA+SVM[20] & 94.23 & $\mathbf{9 4 . 5 8}$ & 96.61 & 95.22 \\
\hline SA+SVM[21] & 97.95 & 87.97 & $\mathbf{9 7 . 5}$ & 91.85 \\
\hline PSO+SVM[22] & 97.95 & 88.17 & $\mathbf{9 7 . 5}$ & 88.32 \\
\hline CV-ACC[23] & 95.97 & 83.98 & 93.68 & 87.26 \\
\hline S.C. Chen [24] & 96.96 & 91.15 & $\mathbf{9 7 . 5}$ & $\mathbf{9 6 . 9 0}$ \\
\hline
\end{tabular}

\section{CONCLUSIONS}

In this paper, we presented a DSA-MSPSVM method for data classification based on MSPSVM and DSA approaches. It is well known that the MSPSVM regularization parameter $\delta$ and kernel parameters are important to the performance of the classifier. But it is difficult to choose a kernel function and its parameters because they are dependent on datasets. The DSA has been applied to optimize these parameters. We conducted experiments to evaluate the performance of the proposed approach with three different kernel functions Poly, RBF, and PolyRBF in the nonlinear classifier. The results obtained were compared with those obtained with other algorithms. The results show enough evidence that the proposed approach has less error rates across most of the datasets with other algorithms. We can also conclude that PolyRBF kernel gives better results as compared with other kernel functions. Further, we plan to extend the DSA-MSPSVM approach to deal with multiclass problems in the linear and nonlinear cases, and study the kernel function effects in the datasets.

\section{REFERENCES}

[1] M.H. Marghny, Rasha M. Abd El-Aziz, and Ahmed I. Taloba. An Effective Evolutionary Clustering Algorithm: Hepatitis C case study. International Journal of Computer Applications, 2011, 34(6): 1-6.

[2] M.H. Marghny, and Ahmed I. Taloba. Outlier Detection using Improved Genetic K-means. International Journal of Computer Applications, 2011, 28(11): 33-36.

[3] Adel A. Sewisy, M.H. Marghny, Rasha M. Abd El-Aziz, and Ahmed I. Taloba. Fast Efficient Clustering Algorithm for Balanced Data. International Journal of Advanced Computer Science and Applications (IJACSA), 2014, 5(6): 123-129.

[4] C.C. Aggarwal, and C.K Reddy. Data clustering: algorithms and applications. Chapman and Hall/CRC Press, 2013.

[5] M.H. Marghny, and I.E. El-Semman. Extracting logical classification rules with gene expression programming: 
microarray case study. Proceedings of the International Conference on Artificial Intelligence and Machine Learning (AIML 05), Cairo, Egypt, 2005, 11-16.

[6] M.H. Marghny, and I.E. El-Semman. Extracting fuzzy classification rules with gene expression programming. Proceedings of the International Conference on Artificial Intelligence and Machine Learning (AIML 05), Cairo, Egypt, 2005.

[7] M.H. Marghny, and H.E. Refaat. A new parallel association rule mining algorithm on distributed shared memory system. International Journal of Business Intelligence and Data Mining, 2012, 7(4): 233-252.

[8] M.H. Marghny, and A.A. Shakour. Fast, Simple and Memory Efficient Algorithm for Mining Association Rules. International Review on Computers \& Software, 2007, 2(1)

[9] M.H. Marghny, and A.A. Shakour. Scalable Algorithm for Mining Association Rules. ICCST, 2006, 6(3): 55-60.

[10] C. Cortes, and V. N. Vapnik. Support vector networks. Machine Learning, 1995, 20(3): 273 - 297.

[11] Glenn Fung, and Olvi L. Mangasarian. Proximal support vector machine classifiers. In Proceedings of the Seventh ACM SIGKDD International conference on knowledge discovery and data mining, KDD 01, ACM, New York, NY, USA, 2001, 77-86.

[12] Olvi L. Mangasarian, and Edward W. Wild. Multisurface proximal support vector machine classification via generalized eigenvalues. IEEE transaction on pattern analysis and machine intelligence, 2006, 28(1): 69-74.

[13] Abe S, and Inoue T. Fuzzy Support Vector Machines for Pattern Classification. IEEE, 2001, 2: 1449-1454.

[14] Chun-Fu Lin, and Sheng-De Wang. Fuzzy support vector machines. IEEE transactions on neural networks, 2002, 13(2): 464-471.

[15] Jayadeva, R. Khemchandani, and S. Chandra. Fuzzy proximal support vector classification via generalized eigenvalues. Springer-Verlag Berlin Heidelberg, 2005, $360-363$

[16] Jayadeva, R. Khemchandani, and S. Chandra. Fuzzy multi-category proximal support vector classification via generalized eigenvalues. Soft Computing - A Fusion of Foundations, Methodologies and Applications, 2007, 11: 679-685.

[17] Ding Shifei, and Gu Yaxiang. A fuzzy support vector machine algorithm with dual membership based on hypersphere, Journal of computational information systems, 2011, 7(6): 2028-2034.

[18] Li. Kai, and Hongyan Ma. A fuzzy twin support vector machine algorithm. International journal of application or innovation in engineering \& management, 2013, 2(3): 459- 465 .

[19] M.R. Guarracino, A. Irpino, R. Jasinevicius, and R. Verde. Fuzzy regularized generalized eigenvalue classifier with a novel membership function. Information Sciences, 2013, 245: 53-62.

[20] Huang. Cheng-Lung, and Chieh-Jen Wang. A ga-based feature selection and parameters optimization for support vector machines. Expert systems with applications, 2006, 31: $231-240$.

[21] S. Lin, Z. Lee, S. Chen, and T. Tseng. Parameter determination of support vector machine and feature selection using simulated annealing approach. Applied soft computing, 2008, 8(4): 1505-1512.

[22] S. Lin, K. Ying, S. Chen, and Z. Lee. Particle swarm optimization for parameter determination and feature selection of support vector machines. Expert systems with applications, 2008, 35(4): 1817-1824.

[23] L. Luo, D. Huang, H. Peng, Q. Zhou, G. Shao, and F. Yang. A new parameter selection method for support vector machine based on the decision value. Convergence information technology, 2010, 5(8): 36-41.

[24] S.C. Chen, S.W. Linb, and S.Y. Chou. Enhancing the classification accuracy by scatter-based ensemble approach. Applied soft computing, 2011, 11(1): 10211028.

[25] X. Zhang, D. Qiu, and F. Chen. Support vector machine with parameter optimization by a novel hybrid method and its application to fault diagnosis. Neurocomputing, 2014, 149(Part B): 641-651.

[26] P. Civicioglu. Understanding the nature of evolutionary search algorithms. Additional technical report for the project of 110Y309-Tubitak, 2013.

[27] D. Goswami, and S. Chakraborty. Differential search algorithm-based parametric optimization of electrochemical micromachining processes. International journal of industrial engineering computations, 2014, 5(1): 41-54.

[28] P. Civicioglu. Transforming geocentric cartesian coordinates to geodetic coordinates by using differential search algorithm. Computers \& Geosciences, 2012, 46: 229-247.

[29] X. Song, L. Li, X. Zhang, X. Shi, J. Huang, J. Cai, S. Jin, J. Ding. An implementation of differential search algorithm (DSA) for inversion of surface wave data. Journal of Applied Geophysics, 2014, 111: 334-345.

[30] R. Devi, E. Barlaskar, O. Devi, S. Medhi, and R. Shimray. Survey on evolutionary computation tech techniques and its application in different fields. International Journal on Information Theory (IJIT), 2014, 3(3): 73-82.

[31] Y. Amrane, M. Boudour, and M. Belazzoug. A new optimal reactive power planning based on Differential Search Algorithm. International Journal of Electrical Power \& Energy Systems, 2015, 64: 551-561.

[32] M.R. Guarracino, C. Cifarelli, O. Seref, and PM. Pardalos. A classification algorithm based on generalized eigenvalue problems. Optimization methods and software, 2007, 22(1): 73-81.

[33] M.R. Guarracino, A. Irpino, and R. Verde. Multiclass generalized eigenvalue proximal support vector machines. Complex, Intelligent and Software Intensive Systems , IEEE Computer Society, 2010, 25-32.

[34] Y. Shao, N. Deng, W. Chen, and W. Zhen. Improved generalized eigenvalue proximal support vector machine. IEEE signal processing letters, 2013, 20(3): 213-216. 
[35] P. Xanthopoulos, M. R. Guarracino, and P. M. Pardalos. Robust generalized eigenvalue classifier with ellipsoidal uncertainty. Ann Oper Res, 2014, 216(1): 327-342.

[36] A.N. Tikhonov, and V.Y. Arsenin. Solutions of Ill posed problems. New York: john wiley and sons, 1977.

[37] B.N. Parlett. The symmetric eigenvalue problem. Philadelphia: SIAM, 1998.
[38] A. Afifi. Improving the classification accuracy using support vector machines (SVMS) with new kernel. Journal of global research in computer science, 2013, 4(2): 1-7.

[39] K. Bache, and M. Lichman. UCI Machine Learning Repository [http://archive.ics.uci.edu/ml]. Irvine, CA: University of California, School of Information and Computer Science, 2013.

\section{APPENDIX}

\section{Pseudo code: Differential search algorithm [28]}

Require:

$\mathrm{N}$ : Size of the population, where $\mathrm{i}=\{1,2,3, \ldots, \mathrm{N}\}$

D: Dimension of the problem

G: Number of maximum generation

1: $\quad$ Superorganism $=$ initialize (), where Superorganism $=\left[\right.$ ArtificialOrganism $\left._{\mathrm{i}}\right]$

2: $\quad \mathrm{y}_{\mathrm{i}}=$ Evaluate $\left(\right.$ ArtificialOrganism $\left.\mathrm{i}_{\mathrm{i}}\right)$

3: $\quad$ for cycle $=1: \mathrm{G}$ do

4: $\quad$ donor $=$ Superorganism Random_Shuffling(i) $_{\text {R }}$

5: $\quad$ Scale $=\operatorname{randg}\left[2 \cdot \operatorname{rand}_{1}\right] .\left(\operatorname{rand}_{2}-\operatorname{rand}_{3}\right)$

6: $\quad$ StopoverSite $=$ Superorganism + Scale.$($ donor - Superorganism $)$

7: $\quad \mathrm{p}_{1}=0.3 \cdot \operatorname{rand}_{4}$ and $\mathrm{p}_{2}=0.3 \cdot \operatorname{rand}_{5}$

8: $\quad$ if $\operatorname{rand}_{6}<\operatorname{rand}_{7}$ then

9: $\quad$ if $\operatorname{rand}_{8}<\mathrm{p}_{1}$ then

10: $\quad r=\operatorname{rand}(\mathrm{N}, \mathrm{D})$

11:

for Counter $1=1: \mathrm{N}$ do

12:

$\mathrm{r}($ Counter $1,:)=\mathrm{r}($ Counter $1,:)<$ rand $_{9}$

13:

end for

14:

else

15:

$\mathrm{r}=$ ones $(\mathrm{N}, \mathrm{D})$

16:

for Counter $2=1: \mathrm{N}$ do

17:

$\mathrm{r}($ Counter $2, \operatorname{randi}(\mathrm{D}))=\mathrm{r}($ Counter $2, \operatorname{randi}(\mathrm{D}))<\operatorname{rand}_{10}$

18:

end for

19:

end if

20: $\quad$ else

21:

22:

$\mathrm{r}=$ ones $(\mathrm{N}, \mathrm{D})$

23:

for Counter $3=1: \mathrm{N}$ do

$$
\mathrm{d}=\operatorname{randi}(\mathrm{D}, 1,[\mathrm{p} 2 \text {.rand } . \mathrm{D}])
$$

$24:$

for Counter4 = 1 : $\operatorname{size}(d)$ do

25:

$$
\mathrm{r}(\text { Counter } 3, \mathrm{~d}(\text { Counter } 4))=0
$$

26:

end for

27:

end for 


\section{8: $\quad$ end if}

29: $\quad$ individuals $_{\mathrm{I}, \mathrm{J}} \leftarrow \mathrm{r}_{\mathrm{I}, \mathrm{J}}>0 \mid \mathrm{I} \in \mathrm{i}, \mathrm{J} \in[1 \mathrm{D}]$

30: $\quad$ StopoverSite $\left(\right.$ individual $\left._{\mathrm{I}, \mathrm{J}}\right):=$ Superorganism(individuals $_{\mathrm{I}, \mathrm{J}}$ )

31: if StopoverSite $e_{i, j}<\operatorname{low}_{i, j}$ or StopoverSite ${ }_{i, j}>$ up $_{i, j}$ then

32: $\quad$ StopoverSite $_{i, j}=\operatorname{rand}^{.}\left(\mathrm{up}_{\mathrm{j}}-\operatorname{low}_{\mathrm{j}}\right)+\operatorname{low}_{\mathrm{j}}$

33: $\quad$ end if

34: $\quad$ y StopoverSite; $\mathrm{i}=$ evaluate $\left(\right.$ StopoverSite $\left._{\mathrm{i}}\right)$

35: $\quad y_{\text {Superorganism } ; i}=\left\{\begin{array}{cl}\mathrm{y}_{\text {StopoverSite } ; \mathrm{i}} & \text { if } \mathrm{y}_{\text {StopoverSite } ; \mathrm{i}}<y_{\text {Superorganism } ; i} \\ y_{\text {Superorganism } ; i} & \text { else }\end{array}\right.$

36: $\quad$ ArtificialOrganism $_{i}= \begin{cases}\text { StopoverSite }_{i} & \text { if } \mathrm{y}_{\text {StopoverSite } ; i}<y_{\text {Superorganism }_{; i}} \\ \text { ArtificialOrganism }_{i} & \text { else }\end{cases}$ 